\title{
PRIMARY RADIATION THERAPY OR SURGERY COMBINED OR NOT TO RADIATION THERAPY IN THE MANAGEMENT OF
} SQUAMOUS CELL CARCINOMA OF THE PENIS

\author{
Abderrahim Zouhair, MD; Mahmut Ozsahin, MD, PhD; Pelham Douglas, MD; Philippe A. Coucke, MD; and René O. Mirimanoff, MD.
}

Department of Radiation Oncology, Lausanne University Medical Center (CHUV), Lausanne, Switzerland.

Purpose: To assess the prognostic factors and the outcome in patients with squamous cell carcinoma of the penis.

Materials \& Methods: A retrospective review of 41 consecutive patients with non-metastatic invasive carcinoma of the penis, treated between 1962 and 1994, was performed. The median age was 59 years (range: $35-76)$. Eight (20\%) patients were circumcised, $30(73 \%)$ were married, and only $2(5 \%)$ had a history of venereal disease. Existence of a penile mass was the first symptom in $32(78 \%)$ patients. The anatomic site was distributed as follows: glans in $17(41 \%)$, prepuce in $9(22 \%)$, shaft in $8(20 \%)$, coronary localization in $4(10 \%)$, prepuce and glans in $2(5 \%)$, and shaft and prepuce in $1(2 \%)$. According to UICC staging, there wcre $12(29 \%) \mathrm{T} 1,24(59 \%) \mathrm{T} 2,4(10 \%) \mathrm{T} 3$, and $1 \mathrm{TX}(2 \%)$ tumors. The $\mathrm{N}$-stagc was distributed as follows: $29(71 \%)$ patients with NO, $8(20 \%)$ with $\mathrm{N} 1,3(7 \%)$ with $\mathrm{N} 2$, and $1(2 \%)$ with N3. Thirteen (32\%) patients had grade 1, 7 grade 2, and 9 grade 3 tumors (grade was determined in 12). Forty-four percent $(n=18)$ of the patients underwent a curative surgery: partial penectomy with $(n=4)$ or without $(n=12)$ lymph node dissection, or total penectomy with $(n=1)$ or without $(n=1)$ lymph node without $(n=12)$ lymph node dissection, or total penectomy with $(n=1)$ or without $(n=1)$ lymph node
dissection. All but 4 parients (operated) underwent primary $(n=23)$ or postoperative $(n=14)$ radiotherapy to the penis and inguinal lymph nodes $(n=20)$, penis alone $(n=9)$, or inguinal lymph nodes alone $(n=8)$. The median and mean follow-up period was 70 and 96 months, respectively (range: $20-331$ ).
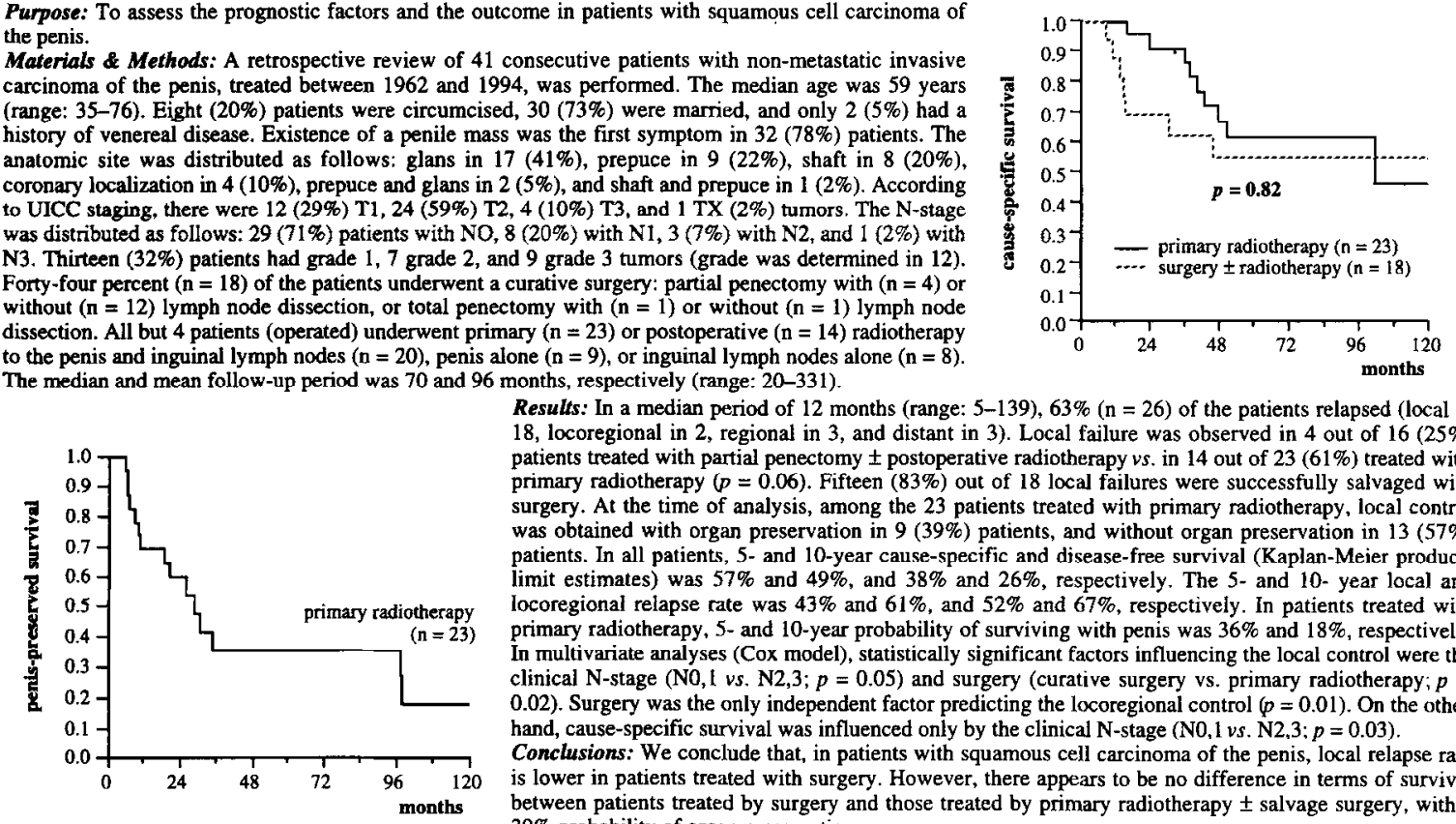

Results: In a median period of 12 months (range: $5-139), 63 \%(n=26)$ of the patients relapsed (local in 18 , locoregional in 2, regional in 3 , and distant in 3). Local failure was observed in 4 out of $16(25 \%)$ patients treated with partial penectomy \pm postoperative radiotherapy vs. in 14 out of 23 (61\%) treated with primary radiotherapy $(p=0.06)$. Fifteen $(83 \%)$ out of 18 local failures were successfully salvaged with surgery. At the time of analysis, among the 23 patients treated with primary radiotherapy, local control was obtained with organ preservation in $9(39 \%)$ patients, and without organ preservation in $13(57 \%)$ patients. In all parients 5- and 10-year cause-specific and disease-free survival (Kaplan-Meier productpimit estimates) was $57 \%$ and $49 \%$, and $38 \%$ and $26 \%$, respectively. The 5- and 10- year local and locoregional relapse rate was $43 \%$ and $61 \%$, and $52 \%$ and $67 \%$, respectively. In patients treated with primary radiotherapy, 5- and 10-year probability of surviving with penis was $36 \%$ and $18 \%$, respectively. In multivariate analyses (Cox model), statistically significant factors influencing the local control were the clinical $\mathrm{N}$-stage (N0,1 vs. $\mathrm{N} 2,3 ; p=0.05$ ) and surgery (curative surgery vs. primary radiotherapy; $p=$ 0.02 ). Surgery was the only independent factor predicting the locoregional control $(p=0.01)$. On the other hand, cause-specific survival was influenced only by the clinical $\mathrm{N}$-stage (N0.1 vs. N2.3: $p=0.03$ ).

Conclusions: We conclude that, in patients with squamous cell carcinoma of the penis, local relapse rate

is lower in patients treated with surgery. However, there appears to be no difference in terms of survival between patients treated by surgery and those treated by primary radiotherapy \pm salvage surgery, with a $39 \%$ probability of organ preservation.

\section{0}

EFFICACY OF ADJUVANT PELVIC AND HIGH DOSE RATE INTRACAVITARY RADIATION IN PATIENTS WTH INTERMEDIATE TO HIGH RISK ENDOMETRIAL CANCER

E.A. Dunphy, 'D.G. Petereit', ${ }^{1,2}$ J.C. Schink', E.A. Grosen ${ }^{2}$, E.M. Hartenbach ${ }^{2}$ and D.A. Buchler ${ }^{1,2}$

University of Wisconsin School of Medicine: 'Department of Human Oncology, 'Department of Gynecology, Madison, Wisconsin 53792

Purpose/Objective: To determine the efficacy of adjuvant whole pelvic radiation (WPRT) in combination with high dose rate (HDR) vaginal cuff brachytherapy (VCB) in patients with intermediate to high risk endometrial cancer.

Material \& Methods: From 4/23/90 to $5 / 6 / 96$, forty-three patients with intermediate to high risk endometrial cancer received adjutvant radiation beginning 4 to 6 weeks after surgery. The following stages of disease were treated: $S$ tage $I B\{1$ pt), Stage IC (7 pts), Stage IIA (10 pts), Stage IIB (15 pts), Stage IIIA $(8 \mathrm{pts})$ and Stage IIIC (2 pts). I'atients had the following histologies: grade 1 adenocarcinoma (t4 pts), grade 2 adenocarcinoma (16 pts), grade 3 adenocarcinoma ( 8 pts); grade 2 adenosquamous carcinoma (1 pt), papillary serous adenocarcinoma (2 pts), papillary serous and clear cell adenocarcinoma ( 1 $\mathrm{pt}$ ) and clear cell adenocarcinoma (1 pt). Patients were treated with WPRT with a median dose of 51 Gy $(1.7 \mathrm{~Gy} / \mathrm{Fx})$, in conjunction with 2 HDR VCB insertions utilizing ovoids where the dose was preserihed to the vaginal surface at 78 Gy/Fx (LDR equivalent of $20 \mathrm{~Gy}$ at $100 \mathrm{cGy} / \mathrm{h}$ ). One patient with Stage IIIA disease had para-aortic radiation to $59.5 \mathrm{~Gy}$ in 35 fractions. Surgery consisted of a TAH/BSO with an assessment of the pelvic lymph nodes in $19 \%$, pelvic/para-aortic lymph nodes in $28 \%$ and /or peritoneal cytology in $37 \%$ of patients. The majority of patients treated in this study had their surgery elsewhere and were referred in for adjuvant radiation. Conplications were scored using the RTOG 5-tiered system. Three year clinical endpoints were calculated using the Kaplan Meier method.

Results: With a median follow-up time of 27 months ( $4-86$ months range), the 3 year survival and relapse-free survival (RFS) were $89 \%$ and $82 \%$, respectively. Two patients recurred in the pelvis: one in the lower $1 / 3$ vagina (4 years after initial treatment) and the second along the entire vagina (both had synchronous distant metastases). One patient developed an isolated para-aortic recurrence. Seven patients had distant recurrences at the following sites: lung and colon (1), lung ankl vagina (2). bone and para-aortics (1). adrenal (1), biliary duct and inguinal lymph nodes (1) and peritoneum (1). All three patients with papillary serous histologies had an upper abdominat recurrence (colon, biliary duct and peritoneum). There was a single grade 3 small bowel complication that was treated medicalty.

Conclusion: Adjuvant whule pelvic and HDR intracavitary radiation produced excellent pelvic control rates (97\% at 3 years) with minimal late toxicities in patients with intermediate to high risk endometrial cancer. More aggressive therapy, such as extended field irradiation, may have reduced the upper abdominal recurrences in patients with papillary scrous (I'S) histologies, but was not delivered because of medical contraindications to whole abdominal radiation. In the other upper abdominal recurrences observed (non-PS histology), extended field radiation may have prevented the development of these sites of disease. Complete surgical evaluation might identify such patients who may benefit from more comprehensive radiation fields. Overall, the excellent pelvic control with a 3 year RFS of $82 \%$ indicates the efficacy of adjuvant radiation for paticnts who have a intermediate to high risk for recurrence without additional therapy. Finally, the vaginal cuff boost with HDR bracliytherapy was well tolerated and did not appear to produce additional complications. The HDR VCB boost may have been partially responsible for the excellent central control rates in this study. 